\title{
Detecting non-Markovianity from continuous monitoring
}

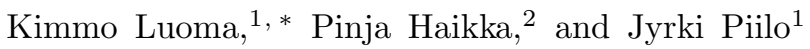 \\ ${ }^{1}$ Turku Centre for Quantum Physics, Department of Physics and Astronomy, \\ University of Turku, FI-20014, Turun Yliopisto, Finland \\ ${ }^{2}$ Department of Physics and Astronomy, Aarhus University, \\ Ny Munkegade 120, DK-8000 Aarhus C, Denmark
}

(Dated: July 30, 2021)

\begin{abstract}
We study how non-Markovianity of an open two-level system can be detected when continuously monitoring a part of its bosonic environment. Considering a physical scenario of an atom in a lossy cavity, we demonstrate that the properties of the time-dependent flux of the photons from the cavity allows the detection of memory effects in the atomic dynamics, without requiring state nor process tomography. This framework overlaps with effective descriptions for the memory part of the environment using pseudomode methods. Our central results show how the Markovian measurement record on the environment of an enlarged open system allows to draw conclusions on the nonMarkovianity of the original system of interest.
\end{abstract}

PACS numbers: 03.65.Yz, 42.50.Lc

\section{INTRODUCTION}

During the last few years, several definitions and quantifiers for non-Markovianity of open quantum system dynamics have been introduced [1]. They are based on a number of different approaches, ranging from concepts of information flow [2, non-divisibility [3] and Fisher information [4 to quantum mutual information [5], accessible volume of physical states [6], channel capacity [7], and kdivisibility [8]. Typically, the experimental detection of memory effects is difficult and requires either process [3] or state tomography [2, 10. Despite recent progresses, developing simple schemes to experimentally detect nonMarkovianity in open system dynamics remains a challenge. In this work we demonstrate how one can detect memory effects in the dynamics of a two-level system by continuously monitoring [11, 12] a part of its environment and keeping the record of the arrival times of the photons.

The evolution of a Markovian open quantum system is governed by the celebrated Gorini-KossakowskiSudarshan-Lindblad master equation 13, 14. This equation can be unravelled with different types of Markovian quantum trajectories [15 18, and one can assign a certain physical reality to these trajectories in a sense that each trajectory represents the state of the open quantum system conditioned on a particular measurement record of some environmental observable [19. The existence of such a measurement scheme interpretation for unravelling of non-Markovian dynamics, instead, has been subject to much debate 20 25. Here we do not make claims about the existence (or non-existence) of the measurement scheme interpretation of conditional pure state evolutions for non-Markovian systems. However, we think that it is important to develop further legitimate schemes

\footnotetext{
* ktluom@utu.fi
}

of measuring the environment of an open system, whether it is Markovian or non-Markovian. In this work we show that a measurement record can, in fact, contain useful information regarding the detection of non-Markovianity without disturbing the open system dynamics. Specifically, we study an analytically solvable system where the environment can be split into a memory and a nonmemory part [26]. If we then monitor the non-memory part of the environment, this does not disturb the underlying non-Markovian dynamics. Similar ideas have been studied recently in Refs. 27 32 .

The outline of this paper is the following. Section II introduces our physical model, Sec. III formulates the main result, and Sec. IV] concludes the Brief Report.

\section{PHYSICAL MODEL}

Our physical system is a two-level atom interacting with a bosonic zero-temperature environment. The Hamiltonian for this system, with $\hbar=1$, is $H=\omega_{A} \sigma_{z}+$ $\sum_{k} \omega_{k} a_{k}^{\dagger} a_{k}+\sum_{k} g_{k}\left(\sigma_{-} a_{k}^{\dagger}+\sigma_{+} a_{k}\right)$, where $\omega_{A}$ is the transition frequency of atom, $\sigma_{z}$ is the Pauli $z$-matrix, $a_{k}$ and $a_{k}^{\dagger}$ are the bosonic annihilation and creation operators for the environmental modes, $g_{k}$ is the coupling between the atom and the $k$-th field mode, and $\sigma_{-}$and $\sigma_{+}$are the lowering and raising operators for the atom. In the continuum limit the sum over the environmental modes can be converted into an integral $\sum_{k} \mapsto \int d \omega_{k} \rho_{k}$, where $\rho_{k}$ is the density of states of the modes.

We focus on a particular physical realization of this model, namely an atom inside a lossy optical cavity, so that the coupling between the atom and the modes $\rho_{k} g_{k}^{2}$ is described by a Lorentzian spectral density $\rho_{k} g_{k}^{2} \equiv$ $J\left(\omega_{k}\right)=\frac{1}{2 \pi} \frac{V^{2} \Gamma}{\left(\omega_{k}-\omega_{c}\right)^{2}+(\Gamma / 2)^{2}}$. Here, $\omega_{c}$ is the cavity resonance frequency, and $\Gamma$ and $V$ describe the width and the strength of the spectral coupling, respectively. If the modes are initially in the vacuum state, it is possible to 
obtain an exact analytical time-local master equation for the two level atom only: $\dot{\rho}(t)=L_{t} \rho(t)$. The analytical form of the superoperator $L_{t}$ is well known, and in certain cases it can describe non-Markovian atomic dynamics [1], 33].

This system can be mapped to a Markovian system $\rho_{\mathrm{AP}}$ with the pseudomode method [34, where the twolevel atom interacts only with a single cavity mode, the pseudomode, which then leaks to a Markovian environment with a constant spectral coupling. The state of the two-level atom is obtained by tracing out the pseudomode $\rho=\operatorname{tr}_{P}\left\{\rho_{\mathrm{AP}}\right\}$ and it obeys exactly the same dynamics as in the original picture. The Markovian master equation for the combined atom and pseudomode system, in the interaction picture with respect to $H_{A}+H_{\mathrm{P}}=\omega_{A} \sigma_{z}+\omega_{\mathrm{P}} a_{\mathrm{P}}^{\dagger} a_{\mathrm{P}}$, is

$$
\begin{aligned}
\dot{\rho}_{\mathrm{AP}}(t)= & -i\left[H_{0}, \rho_{\mathrm{AP}}\right] \\
& -\frac{\Gamma}{2}\left(a_{\mathrm{P}}^{\dagger} a_{\mathrm{P}} \rho_{\mathrm{AP}}+\rho_{\mathrm{AP}} a_{\mathrm{P}}^{\dagger} a_{\mathrm{P}}-2 a_{\mathrm{P}} \rho_{\mathrm{AP}} a_{\mathrm{P}}^{\dagger}\right),
\end{aligned}
$$

where $H_{0}=V\left[e^{i \delta t} \sigma_{-} a_{\mathrm{P}}^{\dagger}+e^{-i \delta t} \sigma_{+} a_{\mathrm{P}}\right], a_{\mathrm{P}}$ and $a_{\mathrm{P}}^{\dagger}$ are the annihilation and creation operators of the pseudomode, $V$ and $\Gamma$ are the spectral coupling and the width of the original system, $\delta=\omega_{\mathrm{P}}-\omega_{A}$, and $H_{A}$ and $H_{\mathrm{P}}$ are the free Hamiltonians of the atom and the pseudomode.

\section{RESULTS}

To solve the Markovian pseudomode master equation (1) we introduce the combined (unnormalised) state $|\tilde{\Psi}(t)\rangle_{\mathrm{AP}}=c_{0}|0\rangle_{A}|0\rangle_{\mathrm{P}}+c(t)|1\rangle_{A}|0\rangle_{\mathrm{P}}+b(t)|0\rangle_{A}|1\rangle_{\mathrm{P}}$ and find the following set of ordinary differential equations

$$
\begin{aligned}
& \dot{c}(t)=-i V e^{-i \delta t} b(t), \\
& \dot{b}(t)=-\frac{\Gamma}{2} b(t)-i V e^{i \delta t} c(t),
\end{aligned}
$$

for the atom and pseudomode amplitudes, respectively, using a technique presented in [34. Coefficient $c_{0}$ is constant throughout the evolution and the analytical solutions of these equations are shown in Appendix A.

The pseudomode, described by operators $a_{\mathrm{P}}$ and $a_{\mathrm{P}}^{\dagger}$, can be interpreted as the memory part of the environment, while the external modes, to which the pseudomode $a_{\mathrm{P}}$ decays, correspond to the non-memory part of the environment [26. Our main result is that we can measure the non-memory part of the environment without disturbing the system of interest and extract enough information from these measurements in order to decide whether the dynamics of the atomic system is Markovian or non-Markovian.

We monitor continuously the photon flux $R(t)$ to the external modes. From the Markovian Monte Carlo wave function (MCWF) method [15] we know that the probability for a quantum jump with jump operator $a_{\mathrm{P}}$ in Eq. (1) during small time interval $[t, t+\delta t)$ is $p(t)=$
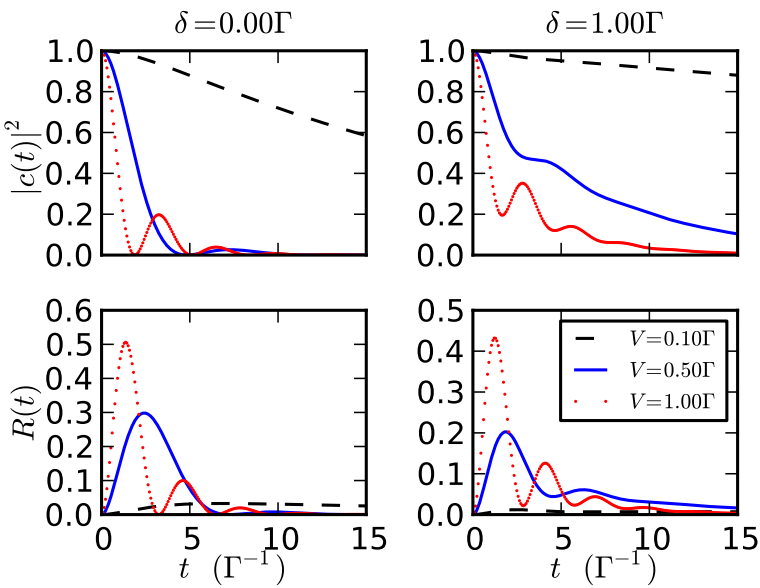

FIG. 1. (color online) Top left: Atom excited state population for different values of $V$ and for $\delta=0 \Gamma$. Top right: Atom excited state population for different values of $V$ and for $\delta=\Gamma$. Bottom left: Photon flux for different values of $V$ and for $\delta=0 \Gamma$. Bottom right: Photon flux for different values of $V$ and for $\delta=\Gamma$. The legend here gives the values of $V$ for all of the panels.

$\delta t \Gamma\left\langle\varphi(t)\left|a_{\mathrm{P}}^{\dagger} a_{\mathrm{P}}\right| \varphi(t)\right\rangle=\delta t R(t)$, where $|\varphi(t)\rangle$ is a single trajectory obtained from the MCWF procedure. This jump corresponds to the emission of a single photon to the external modes. It easy to show that the photon flux from the pseudomode is $R(t)=\Gamma\left\langle 1\left|\operatorname{tr}_{A}\left\{\rho_{\mathrm{AP}}(t)\right\}\right| 1\right\rangle=$ $\Gamma|b(t)|^{2}$, i.e., the product of the decay rate and the population of the pseudomode. Hamiltonian part of Eq. (1) describes the coherent excitation exchange between the atom and the pseudomode. The only way that the excitation can leak out to the external modes is through the pseudomode decay which may occur with probability $p(t)$. After such event the state of the trajectory is $|0\rangle_{A}|0\rangle_{\mathrm{P}}$, and stays there for the rest of the evolution. This means that we can continuously monitor the pseudomode decay without disturbing the non-Markovian dynamics of the atom system.

To detect the photon flux experimentally one needs to be able to prepare the atom in an excited state $c(0)=1$ inside an empty cavity and then detect the times when a photon is emitted from the cavity. Then by repeating the procedure and time-binning the emission times one can construct $R(t)$ experimentally. We denote the total monitoring time with $T$. From now on initial conditions are chosen as $c(0)=1$ and $b(0)=0$. With these fixed initial conditions we can decide if the system is Markovian or non-Markovian for given values of the system parameters (for details see Appendix B).

In Fig. 1. we show the atomic excited state population $|c(t)|^{2}$ and the photon flux $R(t)$ of the pseudomode for different values of coupling $V$ and detuning $\delta$ and for $T=14 / \Gamma$. When $\delta=0$, the atom dynamics is nonMarkovian for $V>\frac{\Gamma}{4}$. In the top left corner one can see oscillations of the excited state populations, a signature 

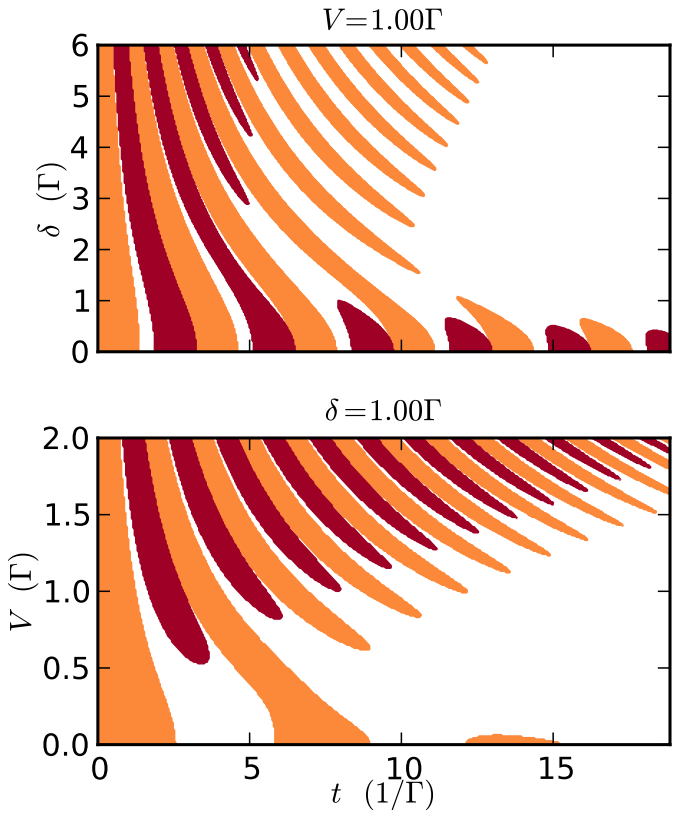

FIG. 2. (color online) Top: Regions in $(t, \delta)$-plane where $C(t)=\partial_{t}|c(t)|^{2}>0$ (dark red, black) and $B(t)=\partial_{t} \Gamma|b(t)|^{2}>$ 0 (orange, gray) for $V=\Gamma$. Bottom: Regions in $(t, V)$-plane for $\delta=\Gamma$ where $C(t)>0$ or $B(t)>0$. Every revival of atomic population $(C(t)>0)$ is followed by increase in the photon flux $(B(t)>0)$. There are also areas where $B(t)>0$ without any previous atomic revivals. For all parameters photon flux increases initially.

of non-Markovian dynamics, for $V=\Gamma / 2$ and $V=\Gamma$. In the bottom left corner one can see oscillations emerging in $R(t)$ for $V=\Gamma$ and very small amplitude oscillations also for $V=\Gamma / 2$. For detuned case $(\delta=\Gamma)$, right column of Fig. 1 atom is again non-Markovian for $V=\Gamma / 2$ and we observe oscillations in the photon flux. The amplitude of $R(t)$ is now smaller since the atom is depleting more slowly. As one can see, whenever the atom dynamics is non-Markovian there are oscillations in the photon flux also. However, oscillating photon flux $R(t)$ is not a sufficient criteria to detect non-Markovianity correctly since $R(t)$ can oscillate when atom dynamics is Markovian if there is sufficient detuning between the atom and the pseudomode transition frequencies (c.f. for example Fig. 1 blue line on right panels).

In Fig. 2 we plot the behavior of $C(t)=\partial_{t}|c(t)|^{2}$ and $B(t)=\partial_{t} \Gamma|b(t)|^{2}$ as a function of time. In the upper panel we have fixed $V=\Gamma$. The dark red (black) areas in the $(t, \delta)$-plane correspond to $C(t)>0$, that is, the atom population is reviving. The orange (gray) areas, on the other hand, correspond to $B(t)>0$, that is an increasing emission rate. In the lower panel we have repeated the calculation with a detuning of $\delta=\Gamma$.

We observe that $B(t)$ is always initially positive, and that if there is a region where $C(t)>0$, this is always followed by a region with $B(t)>0$. This means that the atomic population revival is always followed by an in-

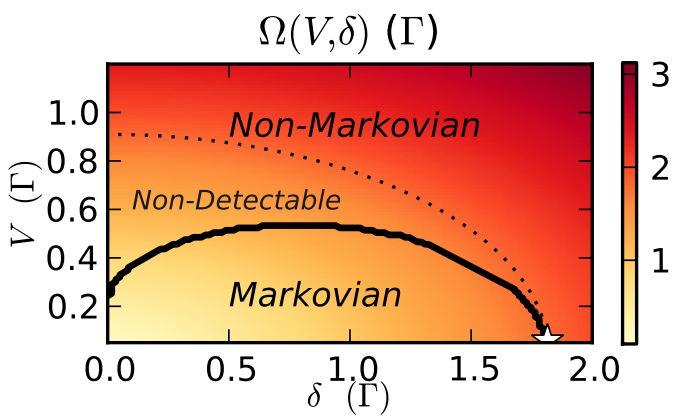

FIG. 3. (color online) Oscillation frequencies $\Omega$ as a function of $V$ and $\delta$ near the transition from Markovian to nonMarkovian dynamics. Below boundary black solid boundary curve dynamics is Markovian. Above Markovian boundary the dynamics of open quantum system is non-Markovian. Maximal Markovian frequency $\Omega_{M} \approx 1.8 \Gamma$ is marked with a white star. With dotted black we plot the contour where, $\Omega(V, \delta)=\Omega_{M}$. Non-Markovianity can not be detected in the region between solid and dotted curves. In this figure $V \in[0.05 \Gamma, 1.2 \Gamma]$.

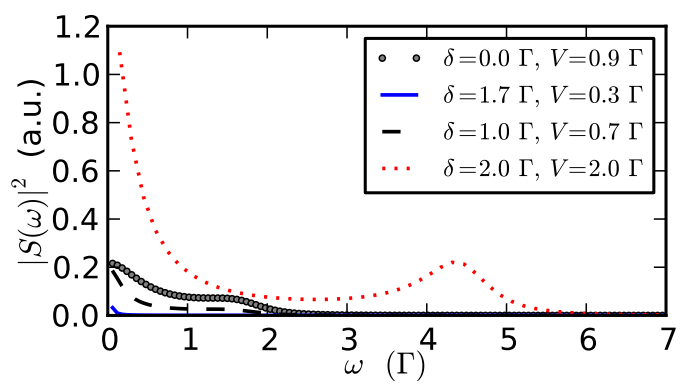

FIG. 4. (color online) Spectrum of the photon flux. $\Omega_{M} \approx$ $1.8 \Gamma$ is the largest Markovian frequency. In this figure we show evidence that for $(\delta, V)$ pair $(2 \Gamma, 2 \Gamma)$ (dotted red line) non-Markovianity is detected because of a pronounced peak in the flux occurring at $\omega \approx 4.47 \Gamma$. Pair $(0.0 \Gamma, 0.9 \Gamma)$ (dotted gray line) and $(\Gamma, 0.7 \Gamma)$ are just below non-detectability border and they show some structure near $\omega \approx \Omega_{M} \cdot(1.7 \Gamma, 0.3 \Gamma)$ (solid blue line) is also near detectability border and in the Markovian region but there is no significant contribution to the spectrum because amplitude of $R(t)$ is very small, but there are oscillations.

crease of the photon flux. However, there are also regions where $B(t)>0$ without preceding areas with $C(t)>0$ (lower panel with $V \approx \Gamma / 2$ ), which means that the oscillating behavior of the photon flux itself is not enough to decide whether the dynamics of the two-level atom is Markovian or non-Markovian.

Still, these findings suggest that non-Markovianity can be detected from the power spectrum $|S(\omega)|^{2}$, which contains information about the oscillation frequencies. We define the spectrum $S(\omega)$ as the Fourier transform of $r(t)=R(t)-\bar{R}_{T}$, where $\bar{R}_{T}=\frac{1}{T} \int_{0}^{T} R(s) \mathrm{d} s$ is the timeaverage of the photon flux and $T$ is the total time of 
observation. The Wiener-Khinchin theorem states that the power spectrum is related to the auto-correlation function $\kappa(t)=\int_{-\infty}^{\infty} \mathrm{d} \tau r(\tau) r(\tau+t)$ through the inverse Fourier transform (for more details, see Appendix C). It turns out that, when effect of damping is negligible, then there are periodic oscillations in the photon flux $R(t)$. The frequency of these oscillations is

$$
\Omega \equiv \Omega(V, \delta)=\sqrt{4 V^{2}+\delta^{2}}
$$

Numerical evidence shows that the effect of damping $\Gamma$ is negligible to the oscillation frequency in the nonMarkovian regime for short times. In the Markovian regime, the oscillation is damped after couple of cycles, has a small amplitude and is not periodic, hence it does not contribute to the power spectrum. In other words, when atom and pseudomode are coupled strongly enough, relative to the damping, there is coherent oscillation that can be observed in the spectrum and this occurs in the non-Markovian parameter regime. Therefore using $\Omega$ and calculating maximum frequency $\Omega_{M}$ in the Markovian parameter region, we obtain a threshold frequency that tells us that if there is coherent oscillations in the atom pseudomode dynamics with $\Omega>\Omega_{M}$, the atom dynamics is non-Markovian.

In Fig. 3 we show $\Omega(V, \delta)$ as a density plot with a boundary curve (solid black) between the Markovian and non-Markovian regions in parameter space and with a contour curve $\Omega(V, \delta)=\Omega_{M}$ (dashed black). We have also denoted the maximal Markovian frequency $\Omega_{M} \approx$ $1.8 \Gamma$ with a white star. Between Markovian boundary curve and contour curve corresponding to $\Omega_{M}$ there is region of ambiguity where non-Markovianity could not be reliably detected. This occurs because Markovian dynamics could, in principle, contribute to the power spectrum with these frequencies.

In Fig. 4 we study the behavior of the power spectrum $|S(\omega)|^{2}$. We have calculated the spectrum in all three regions of Fig. 3. Solid blue curve $(\delta, V)=(1.7 \Gamma, 0.3 \Gamma)$ is in the Markovian region, dashed black $(\Gamma, 0.7 \Gamma)$ and dotted gray curves $(0,0.9 \Gamma)$ are in the non-detectable region and dotted red curve is in non-Markovian detectable region $(2 \Gamma, 2 \Gamma)$. In this figure we see that when the parameters are such that we are outside of the non-detection region we have a pronounced peak in the emission spectrum. We have also studied the behavior of the Markovian boundary near the non-detectability/non-Markovian boundary (solid blue curve) and it shows that choosing the threshold as we have done excludes false positive detections of non-Markovianity. What can also be deduced is that the pronounced behavior of the red dotted curve can be seen only outside non-detection region when the open system exhibits non-Markovian dynamics. This figure confirms that threshold frequency $\Omega_{M}$ is compatible with non-Markovianity of the atom dynamics.

\section{SUMMARY}

To summarize, we have demonstrated that it is possible to detect the presence of memory effects for an open two-level system without doing a full state tomography - provided that its non-Markovian dynamics is not perturbed by measurements on the environment. This was shown by mapping the original non-Markovian system to a larger Markovian one, where the memory and the nonmemory parts of the environment can be identified. For this purpose, we considered a two-level atom in a lossy cavity as a concrete example.

In the original system, non-Markovianity is caused by the strong coupling between the atom and bosonic modes which is manifested in the oscillations of the excited state population. In the extended atom and single cavity mode system, the atom and the cavity mode may exchange excitation coherently many times before the cavity mode is damped. This information is then encoded into the flux of the emitted photons from the cavity and its power spectrum. As we showed, this information within the environment of the extended Markovian system allows us also to detect the presence of memory effects in the original open two-level system.

Our results are based on an interplay between the oscillations of the atomic excited state population and the flux of emitted photons from a cavity. It is expected that in the non-Markovian region the oscillations in the atomic excited state population also show up as oscillations in the photon flux from the cavity. However, in general the situation is more complicated since the time dependent photon flux may also oscillate in the Markovian region when the atomic excited state population decreases monotonically. Subsequently, we have presented a spectrum analysis of the photon signal which allows to detect the presence of memory effects without directly measuring the state of the atom. In other words, there exists a threshold value in the frequency of the time dependent photon flux oscillations which allows to study the Markovian and non-Markovian regions for the two-level atom dynamics. The experimental implementation of the scheme presented here is realistic with an ion trapped inside a cavity 35 .

\section{ACKNOWLEDGMENTS}

KL and JP would like to thank Vilho, Yrjö, and Kalle Väisälä Foundation, and Jenny and Antti Wihuri Foundation for financial support. PH acknowledges funding from the European Comission (ITN CCQED). This research was undertaken on Finnish Grid Infrastructure (FGI) resources. 


\section{Appendix A: Analytical solution for $c(t)$ and $b(t)$}

Solutions to Eqs. (2)- (3) with initial condition $b(0)=0$ for cavity mode and generic initial condition $c(0)$ for the excited state amplitude of the atom

$$
\begin{aligned}
c(t) & =e^{-\frac{1}{4} t(\Gamma+2 i \delta)} c(0)\left(\cosh \left(\frac{d t}{4}\right)+\frac{\Gamma+2 i \delta}{d} \sinh \left(\frac{d t}{4}\right)\right), \\
b(t) & =\frac{2 i e^{-\frac{1}{4} t(d+\Gamma-2 i \delta)}\left(e^{\frac{d t}{2}}-1\right) V c(0)}{d} \\
d & =\sqrt{-16 V^{2}+(\Gamma+2 i \delta)^{2}}
\end{aligned}
$$

Solutions are obtained by Laplace transform method.

\section{Appendix B: Measure for non-Markovianity}

According to the reference [2], the dynamical map $\Phi_{t}$ describes non-Markovian evolution, if for some initial pair of states $\rho_{1,2}(0)$, their trace distance $D\left(\rho_{1}(t), \rho_{2}(t)\right)=\frac{1}{2} \operatorname{tr}\left|\rho_{1}(\mathrm{t})-\rho_{2}(\mathrm{t})\right|$ increases temporally $\sigma(t)=\partial_{t} D\left(\rho_{1}(t), \rho_{2}(t)\right)>0$. Measure for nonMarkovianity is then obtained by

$$
\mathcal{N}=\max _{\rho_{1,2}(0)} \int_{\sigma(t)>0} \sigma(t) .
$$

For our scheme, we want to obtain as large as possible photon flux to the external modes of the extended
Markovian system. This is achieved when initially the atom is maximally excited $\rho_{\mathrm{AP}}(0)=|1\rangle\langle 1|\otimes| 0\rangle\langle 0|$ and $\rho_{1}(0)=|1\rangle\langle 1|$. Maximizing pair for $\mathcal{N}$ must be orthogonal [36], which leads in the considered purpose $\rho_{2}(0)=|0\rangle\langle 0|$ and the trace distance is equal to the excited state population of $\rho_{1}(t)$

$$
D\left(\rho_{1}(t), \rho_{2}(t)\right)=\left\langle 1\left|\rho_{1}(t)\right| 1\right\rangle=|c(t)|^{2} .
$$

For the current system, at least the divisibility measure [3] and the above trace distance measure [2] are compatible since there is only one decay channel and the dynamics becomes non-Markovian when the corresponding decay rate becomes temporarily negative.

\section{Appendix C: Spectrum $S(\omega)$}

Let $r\left(t_{i}\right)=r_{i} \in \mathbb{R}$ be our experimental signal sampled at intervals $\delta t$ apart, $t_{i}=i \delta t, i=[0, N-1]$. Let sampling rate be $f_{s}$ and $\delta t=\frac{1}{f_{s}}$. Discrete (only positive) frequencies are then $\frac{i f_{s}}{N}$, where $i \in[0, N / 2-1]$ for $N$ even and $i \in[0,(N-1) / 2]$ for $N$ odd. Fourier transform of discrete signal is defined as $S\left(\omega_{k}\right)=S_{k}=$ $\sum_{m=0}^{N-1} r_{m} e^{-2 \pi i m k / N}$. Inverse Fourier transform is defined as $r_{m}=\frac{1}{N} \sum_{k=0}^{N-1} S_{k} e^{2 \pi i m k / N}$. This leads to the following normalization $\sum_{m}\left|r_{m}\right|^{2}=\frac{1}{N} \sum_{k}\left|S_{k}\right|^{2}$ (Parseval's theorem). We have also the following relation $\sum_{m} r_{m} r_{m+k}=\frac{1}{N} \sum_{l}\left|S_{l}\right|^{2} e^{2 \pi i k l / N}$ (Wiener-Kinchin theorem).
[1] M. M. Wolf, J. Eisert, T. S. Cubitt, and J. I. Cirac, Phys. Rev. Lett. 101, 150402 (2008).

[2] H.-P. Breuer, E.-M. Laine, and J. Piilo, Phys. Rev. Lett. 103, 210401 (2009)

[3] A. Rivas, S. F. Huelga, and M. B. Plenio, Phys. Rev. Lett. 105, 050403 (2010).

[4] X. M. Lu, X. Wang, and C. P. Sun, Phys. Rev. A 82, 042103 (2010).

[5] S. Luo, S. Fu, and H. Song, Phys. Rev. A 86, 044101 (2012)

[6] S. Lorenzo, F. Plastina, and M. Paternostro, Phys. Rev. A 88, 020102(R) (2013).

[7] B. Bylicka, D. Chruściński, and S. Maniscalco, Sci. Rep. 4, 5720 (2014).

[8] D. Chruściński and S. Maniscalco, Phys. Rev. Lett. 112, 120404 (2014)

[9] F. Buscemi and N. Datta, ArXiv e-prints (2014), arXiv:1408.7062 [quant-ph].

[10] B.-H. Liu, L. Li, H. Y.-F., C.-F. Li, G.-C. Guo, E.-M. Laine, H.-P. Breuer, and J. Piilo, Nature Physics 7, 931 (2011).

[11] H. P. Breuer and F. Petruccione, The theory of open quantum systems (Oxford University Press, Oxford, 2002).

[12] H. Carmichael, Statistical Methods in Quantum Optics 2: Non-Classical Fields, Statistical Methods in Quantum
Optics (Springer, 2007).

[13] V. Gorini, A. Kossakowski, and E. C. G. Sudarshan, Journal of Mathematical Physics 17, 821 (1976).

[14] G. Lindblad, Communications in Mathematical Physics 48, 119 (1976)

[15] J. Dalibard, Y. Castin, and K. Mølmer, Phys. Rev. Lett. 68, 580 (1992).

[16] C. W. Gardiner, A. S. Parkins, and P. Zoller, Phys. Rev. A 46, 4363 (1992).

[17] W. T. Strunz, L. Diósi, and N. Gisin, Phys. Rev. Lett. 82, 1801 (1999)

[18] T. Yu, L. Diósi, N. Gisin, and W. T. Strunz, Phys. Rev. A 60, 91 (1999).

[19] H. M. Wiseman and G. J. Milburn, Phys. Rev. A 47, 642 (1993).

[20] L. Diósi, N. Gisin, and W. T. Strunz, Phys. Rev. A 58, 1699 (1998).

[21] M. W. Jack and M. J. Collett, Phys. Rev. A 61, 062106 (2000)

[22] J. Gambetta and H. M. Wiseman, Phys. Rev. A 66, 012108 (2002)

[23] J. Piilo, S. Maniscalco, K. Härkönen, and K.-A. Suominen, Phys. Rev. Lett. 100, 180402 (2008).

[24] L. Diósi, Phys. Rev. Lett. 100, 080401 (2008).

[25] H. M. Wiseman and J. M. Gambetta, Phys. Rev. Lett. 101, 140401 (2008) 
[26] L. Mazzola, S. Maniscalco, J. Piilo, K.-A. Suominen, and B. M. Garraway, Phys. Rev. A 80, 012104 (2009).

[27] H.-P. Breuer, Phys. Rev. A 75, 022103 (2007)

[28] L. Diósi, Phys. Rev. A 85, 034101 (2012).

[29] H. Yang, H. Miao, and Y. Chen, Phys. Rev. A 85, 040101 (2012)

[30] A. A. Budini, Phys. Rev. A 88, 012124 (2013)
[31] A. Shabani, J. Roden, and K. B. Whaley, Phys. Rev. Lett. 112, 113601 (2014)

[32] T. Ma, Y. Chen, T. Chen, S. R. Hedemann, and T. Yu, ArXiv e-prints (2014), arXiv:1404.5280 [quant-ph].

[33] J.-G. Li, J. Zou, and B. Shao, Phys. Rev. A 81, 062124 (2010)

[34] B. M. Garraway, Phys. Rev. A 55, 2290 (1997)

[35] M. Keller and H. Takahashi, private communication.

[36] S. Wißmann, A. Karlsson, E.-M. Laine, J. Piilo, and H.-P. Breuer, Phys. Rev. A 86, 062108 (2012). 